\title{
ON THE R-MATRIX FORMULATION OF DEFORMED ALGEBRAS AND GENERALIZED JORDAN-WIGNER TRANSFORMATIONS
}

\author{
S.Meljanac ${ }^{1}$, M.Mileković ${ }^{2,+}$ and A.Perica ${ }^{1,++}$ \\ ${ }^{1}$ Rudjer Bošković Institute, Bijenička c.54, 41001 Zagreb \\ Croatia \\ ${ }^{2}$ Prirodoslovno-Matematički Fakultet, Department of Theoretical Physics, \\ Bijenička c.32, 41000 Zagreb \\ Croatia \\ + e-mail:marijan@phy.hr \\ ++ e-mail: perica@thphys.irb.hr
}

Classification number: $02.20+b ; 03.65$ 


\begin{abstract}
The deformed algebra $\mathcal{A}(\mathcal{R})$, depending upon a Yang-Baxter R- matrix, is considered. The conditions under which the algebra is associative are discussed for a general number of oscillators. Four types of solutions satisfying these conditions are constructed and two of them can be represented by generalized Jordan-Wigner transformations.Our analysis is in some sense an extension of the boson realization of fermions from single-mode to multimode oscillators.
\end{abstract}


The introduction of q-deformations of the Heisenberg-Weyl algebras [1] has led to the investigation of particles obeying the statistics different from the ordinary Bose and Fermi statistics, referred to as q-bosons and q-statistics,respectively. Two particularly useful formulations of the q-deformed associative Heisenberg-Weyl algebra are proposed through (i) constant solutions of the Yang-Baxter-Hecke equations (R-matrices), which generalize the notion of permutational symmetry [2], and (ii) generalized Jordan-Wigner mappings from the undeformed Bose algebra [3], resembling bosonization techniques widely used in nuclear physics [4] and other fields of theoretical physics [5]. These two approaches may be considered as complementary in the sense that there exist q-algebras which can be formulated in terms of R-matrices but not as regular mappings from the Bose algebra, and vice versa.

In a recent paper [6] the investigation was started with the algebra of covariant Pusz-Woronovicz oscillators and the corresponding $S U(n)_{q}$ R-matrix formulation of the deformed Bose algebra. Then, the same form of deformed algebras for a general R-matrix was postulated. Under the requirements of associativity and hermiticity, a set of conditions ( the Yang-Baxter and the Hecke equations ) was obtained. These equations were solved for two oscillators using an ansatz that the R-matrices are of the eight-vertex type, and three types of solutions were found.

In this paper we construct four classes of deformed algebras for the general case of $n$ oscillators with the corresponding R-matrices. The main characteristic of these algebras is whether or not they can be obtained by continuous mapping from the Bose algebra.This can be viewed as a generalization of the boson realization of fermions to multimode oscillators of various types [7]. 
Let us start with the R-matrix deformed algebra [6] for n oscillators

$$
\begin{gathered}
a_{i} a_{j}-p R_{i j, k l} a_{l} a_{k}=0 \\
a_{i} a_{j}^{+}-p^{\prime} R_{k i, j l} a_{k}^{+} a_{l}=\delta_{i j}
\end{gathered}
$$

where $\mathrm{p}$ and p' are real parameters and $(i, j, k, l) \epsilon(1,2, \ldots . n)$. The associativity and hermiticity requirements lead to the following conditions:

(A) the Yang-Baxter equation

$$
\sum_{u, v, w} R_{a b, u v} R_{v w, c d} R_{u e, f w}=\sum_{u, v, w} R_{b e, u v} R_{w u, f c} R_{a v, w d}
$$

(B) the Hecke condition

$$
(p \hat{R}-1)\left(p^{\prime} \hat{R}+1\right)=0
$$

where $\hat{R}=\mathrm{PR}$ and $\mathrm{P}$ is the permutation operator $P_{i j, k l}=\delta_{j k} \delta_{i l}$, and the hermiticity requirement is $R_{i j, k l}=R_{l k, j i}^{*}$ or $\hat{R}^{+}=\hat{R}$.

For general n, there are two types of solutions given by

$$
p \hat{R}=1 \quad \text { i.e. } \quad p R=P
$$

with the corresponding algebra

$$
a_{i} a_{j}^{+}=Q \delta_{i j}
$$

where

$$
Q=1+\frac{p^{\prime}}{p} \sum_{i=1}^{n} a_{i}^{+} a_{i}
$$


(ii) $\quad p^{\prime} \hat{R}=-1 \quad$ i.e. $\quad p^{\prime} R=-P$

with the corresponding algebra

$$
\begin{gathered}
a_{i} a_{j}^{+}=Q \delta_{i j} \\
a_{i} a_{j}=0
\end{gathered}
$$

where

$$
Q=1-\sum_{i=1}^{n} a_{i}^{+} a_{i}
$$

The main characteristic of solutions (i) and (ii) is that there exists no mapping to the Bose algebra. In case (i) there are no commutation relations between the annihilation $a_{i}, a_{j}$ ( creation $a_{i}^{+}, a_{j}^{+}$) operators. The $a_{i}, a_{j}$ operators behave as some kind of generalized quons and, in the limit $\frac{p^{\prime}}{p}=0$, they are the same as quons with $q=0$ [8]. In case (ii), the oscillators behave as objects with the hard-core condition $a_{i}^{2}=0$. This is in some sense similar to the fermionic algebra. The algebra (4) is described by Klein and Marshalek in refs. [4] [9].

In addition to solutions (i) and (ii) there are other two types of solutions corresponding to oscillators that can be obtained by mappings from Bose oscillators in the form of generalized Jordan-Wigner transformations.

(iii) One mapping leading to the q-deformed algebra (1) is of the form

$$
a_{i}=\left[\prod_{j=1}^{n}\left(\kappa_{i j} q\right)^{\theta_{i j} N_{j}}\right] b_{i} \sqrt{\frac{\left[N_{i}\right]_{\chi_{i}}}{N_{i}}}
$$


where

$$
\begin{gathered}
{\left[N_{i}\right]_{\chi_{i}}=\frac{\chi_{i}^{N_{i}}-1}{\chi_{i}-1}} \\
\chi_{i}=\frac{q^{2}-1}{2}+\epsilon_{i} \frac{q^{2}+1}{2} \\
\kappa_{i j}=\kappa_{j i} \in\{-1,1\} \\
\epsilon_{i} \in\{-1,1\}, \quad q \in \mathbf{R}^{+}
\end{gathered}
$$

and $N_{i}$ is the number operator for the i-th oscillator mode.

The corresponding deformed algebra is explicitly given by

$$
\begin{gathered}
\left(\epsilon_{i}-1\right) a_{i}^{2}=0 \\
a_{i} a_{j}=\kappa_{i j} q^{s g n(j-i)} a_{j} a_{i} \\
a_{i} a_{j}^{+}=\kappa_{i j} q a_{j}^{+} a_{i}, \quad i \neq j \\
a_{i} a_{i}^{+}=q^{2 \sum_{j} \theta_{i j} N_{j}}+\chi_{i} a_{i}^{+} a_{i}= \\
=1+\left(q^{2}-1\right) \sum_{j} \theta_{i j} a_{j}^{+} a_{j}+\chi_{i} a_{i}^{+} a_{i}
\end{gathered}
$$

where $\theta_{i j}$ is a step-function.

The corresponding R-matrix is of the form

$$
p^{\prime} R=\sum_{i} \chi_{i} e_{i i} \otimes e_{i i}+q \sum_{i \neq j} \kappa_{i j} e_{i i} \otimes e_{j j}+\left(q^{2}-1\right) \sum_{i<j} e_{i j} \otimes e_{j i}=q^{2} p R
$$

where $\left(e_{i j}\right)_{k l}=\delta_{i k} \delta_{j l}$.

In solution (iii) there are ( in addition to ordinary bosons, fermions and Green's oscillators for para-Bose and para-Fermi statistics [10] ) covariant Pusz-Woronowicz oscillators of both the bosonic and the fermionic type. This is a generalization of 
the results in ref. [7] to multimode oscillators. The algebra in eq.(8) is automatically associative and norms of the states in the Fock space are positive definite.

(iv) There is also another mapping leading to the q-deformed algebra (1):

$$
a_{i}=e^{i \sum_{j}\left(\lambda_{i j}-\lambda_{j i}\right) N_{j}} b_{i} \sqrt{\frac{\left[N_{i}\right]_{\xi_{i}}}{N_{i}}}
$$

with $\lambda_{i j}$ real numbers and $\xi_{i}= \pm 1$. The oscillators $a_{i}$ are of the anyonic type in the single-valued picture in $(2+1)$ dimensions, where $\lambda_{i j}$ becomes the angle function [3] 11] 12]. They satisfy the following algebra:

$$
\begin{gathered}
\left(\xi_{i}-1\right) a_{i}^{2}=0 \\
a_{i} a_{j}=e^{i\left(\lambda_{j i}-\lambda_{i j}\right)} a_{j} a_{i} \quad i \neq j \\
a_{i} a_{j}^{+}=e^{i\left(\lambda_{i j}-\lambda_{j i}\right)} a_{j}^{+} a_{i}, \quad i \neq j \\
a_{i} a_{i}^{+}=1+\xi_{i} a_{i}^{+} a_{i}
\end{gathered}
$$

$\xi_{i}=1\left(\xi_{i}=-1\right)$ corresponds to the Bose - ( Fermi- ) type commutation relation. The above algebra can be written in the R-matrix formulation,eq.(1), only for $\xi_{i}= \pm 1$

Then,the corresponding R-matrix is

$$
p^{\prime} R=\sum_{i} \xi_{i} e_{i i} \otimes e_{i i}+\sum_{i \neq j} e^{i\left(\lambda_{i j}-\lambda_{j i}\right)} e_{i i} \otimes e_{j j}=p R
$$

or

$$
(p R)_{i j, k l}=e^{i\left(\lambda_{i j}-\lambda_{j i}\right)} \delta_{i k} \delta_{j l}+\left(\xi_{i}-1\right) \delta_{i j} \delta_{i k} \delta_{j l}
$$

This R-matrix can be written in the continuum limit leading to the q-deformed field theory in $(2+1)$ dimensions [11]. Pure anyons are characterized by $\lambda_{i j}-\lambda_{j i}=$ 
$\lambda \pi \operatorname{sgn}(i-j)$, with $\lambda=$ const. We point out that multivalued anyons in $2+1$ dimensions [13] can also be written in the form of multivalued mapping to bosons [14] and the corresponding algebra is given by

$$
\begin{gathered}
a_{i} a_{j}=e^{i \lambda \Delta} a_{j} a_{i} \\
a_{i} a_{j}^{+}=\delta_{i j}+e^{i \lambda \Delta} a_{j}^{+} a_{i} \\
\Delta=\{\pi(1+2 z) ; z \in Z\}
\end{gathered}
$$

and the R-matrix is

$$
(p R)_{i j, k l}=\left(p^{\prime} R\right)_{i j, k l}=e^{i \lambda \Delta} \delta_{i k} \delta_{j l}
$$

where the R-matrix elements are multivalued numbers. ( The multiplication "unit" is of the form $\left.E=e^{2 i k \pi \lambda}, k \in \mathbf{Z}\right)$.

Our solution (iv) of the anyonic type for $n=2$ was overlooked in [6]. Adding this solution to the solutions in [6] makes the list of solutions for $n=2$ complete.This can be checked by inspection of the complete list of solutions to the Yang-Baxter equations for $n=2[15]$. Finally, there may exist other solutions with "peculiar" statistics for which no mapping to the Bose algebra exists ( for $n=2$ see [6] ) and the number operators do not exist in the usual sense. Owing to their "peculiarity",it is interesting to discuss the Fock-space representation of "peculiar" algebras and to construct them for a general number of oscillators. The results of this investigation are reported elsewhere [16]. 


\section{Acknowledgments}

This work was supported by the joint Croatian-American contract NSF JF 999 and the Scientific Fund of the Republic of Croatia. 


\section{References}

[1] Biedenharn L.C., it J. Phys.A :Math.Gen.22 (1989) L873;

Macfarlane A.J., J. Phys.A :Math.Gen.22 (1989) L983;

Pusz W. and Woronowicz S. L., Rep.Math.Phys. 27 (1989) 231;

Hayashi T., Comm.Math.Phys. 127 (1990) 129;

Chaichian M. and Kulish P.P., Quantum superalgebras,q-oscillators and applications, preprint CERN-TH.5969/90;

Meljanac S.,Mileković M. and Pallua S., Unified view of deformed single-mode oscillator algebra,preprint HEP-TH-9404039 (to appear in Phys.Lett.B).

[2] Fadeev L.D.,Reshetikhin N.Y. and Takhtajan L.A., in Algebraic Analysis, ed. M.Kashiwara and T.Kawai, (Academic Press,N.Y.,1989);

Kulish P.P., Phys.Lett.161A (1991) 50;

Kempf A., J.Math.Phys.34 (1993) 969.

[3] Fairlie D. and Zachos C., Phys.Lett. 256B (1991) 43.

[4] Klein A. and Marshalek E.R., Rev.Mod.Phys.63 (1991) 375.

[5] Huerta L. and Zanelli J., Phys.Rev.Lett.71 (1993) 3622;

Marchetti P.A., Nucl.Phys.B Proc.Supll.33C (1993) 134 and references therein.

[6] Van der Jeugt J., J. Phys.A :Math.Gen. 26 (1993) L405.

[7] Ruan T.,Jing S. and Wang A., Europhys.Lett.23 (1993) 317;

Kuchta R. and Takada K., Europhys.Lett.25 (1993) 319.

[8] Greenberg O. W. Phys.Rev.Lett. 64 (1990) 705 ; Phys.Rev.D 43 (1991) 4111. 
[9] Klein A. and Marshalek E.R., Z.Phys.A329 (1988) 441.

[10] Green H. S., Phys.Rev.90 (1953) 270;

Ohnuki Y. and Kamefuchi S., Quantum Field Theory and Parastatistic ( University of Tokio Press, Tokio, Springer, Berlin, 1982 )

[11] Bardek V., Dorešić M. and Meljanac S., Int.J.Mod.Phys..A9 (1994) 4185.

[12] Lerda A. and Sciuto S., Nucl.Phys.B 401 (1993) 613.

[13] Leinaas J. M. and Myrheim J., Nuovo Cim.B 37 (1977) 1;

Wilczek F., Phys.Rev.Lett. 48 (1982) 1144; ibid. 49 (1982) 957.

[14] Bardek V., Dorešić M. and Meljanac S., Phys.Rev.D 49 (1994) 3059.

[15] Hietarinta J., Phys.Lett, A 165 (1992) 245.

[16] Meljanac S.,Mileković M. and Perica A., A new deformed supersymmetric oscillator, preprint RBI-TH-6/94 (submitted to J.Phys.A:Math.Gen.). 